Ann. Biol. anim. Bioch. Biophys., I970, 10 (2), 313-316.

NOTE

\title{
ACTIVATION DES ENZYMES LYSOSOMALES DU MUSCLE AU COURS DE LA MATURATION DE LA VIANDE
}

\author{
C. VALIN \\ avec la collaboration technique de A. OBLED \\ Station de Recherches sur la Viande, \\ Centre de Recherches de Clermont-Ferrand, 63-Saint-Genès-Champanelle \\ Institut national de la Recherche agronomique
}

L'implication des enzymes lysosomales du muscle dans les mécanismes biochimiques de la maturation de la viande a été l'objet de nombreuses controverses (LAWRIE, I966). Exprimés en terme ultime de dégradation des protéines c'est-à-dire en acides aminés libérés, tous les résultats concordent pour conclure à un faible niveau d'activité protéolytique au cours de la maturation (Gardner et SteWart, I966; SuzUkI et al., I967). Au niveau des éléments constitutifs de la structure du muscle, aucune attaque protéolytique des protéines myofibrillaires n'a pu être mise en évidence (Bodwell, Pearson, I964; Martins, Whitaker, i968) ; par contre, pour des durées de maturation supérieures à huit jours McInTosh (1967) a pu montrer une évolution des mucoprotéines du tissu interstitiel analogue à celle produite in vitro par la papaïne sur ces composés.

Parallèlement à ces investigations au niveau des substrats la détermination des activités hydrolytiques libres in situ permettrait de préciser les mécanismes mis en jeu. L'activation des enzymes lysosomales est fonction de leur libération, donc de la destruction post mortem des structures auxquelles elles sont liées. S'il est aisé d'étudier in vitro sur des suspensions de lysosomes purifiés (SAWANT et al., 1964) les conditions physiques et l'action d'agents chimiques susceptibles d'entraîner la libération de ces enzymes, il s'avère difficile d'extrapoler les résultats, obtenus en général sur des préparations de lysosome de foie de rat, à d'autres tissus (RAHMAN, I964). De plus, la grande fragilité des membranes des lysosomes entraîne une libération importante d'enzymes lors de l'homogénéisation des tissus, ceci étant d'autant plus sensible lorsqu'il s'agit du muscle. Les activités libres mesurées dans ces conditions risquent d'être très supérieures à l'activité libre réelle in situ. Il conviendrait donc pour effectuer la détermination des activités libres dans le tissu musculaire d'éviter tout traitement mécanique brutal lors de l'extraction des protéines. Dans ce but et compte tenu des travaux de AMBERSON et al. (1964) et de SAWANT et al. (1964) nous avons procédé à l'ultracentrifugation de fragments de muscle en milieu légèrement hypertonique. Cette technique permet l'extraction partielle des protéines sarcoplasmiques du muscle sans provoquer de libération des enzymes lysosomales.

L'étude a été effectuée sur le muscle Sternocephalicus de bovin. Les prélèvements ont été opérés immédiatement après l'abattage, 2 jours et 8 jours post mortem. Les carcasses étaient conservées en chambre froide à $+4^{\circ} \mathrm{C}$. 
L'extraction des protéines sarcoplasmiques est effectuée en deux temps. Une première phase d'imbibition de 2 heures à $+4^{\circ} \mathrm{C}$ dans une solution de saccharose $0,45 \mathrm{M}$, EDTA ${ }^{-3}{ }^{-3} \mathrm{M}$, tampon tris- $\mathrm{HCl} 0,02 \mathrm{~N} \mathrm{pH} 7,4$ de petits fragments de muscle (environ I g) découpés au scalpel, puis ultracentrifugation des fragments de muscle dans le milieu d'imbibition pendant 150 minutes à I65 ooo g à la température de $0^{\circ} \mathrm{C}$ (Spinco L 50 rotor $50 \mathrm{TI}$ ).

Les protéines sarcoplasmiques qui ont diffusé dans la solution de saccharose et celles qui ont été exprimées du muscle au cours de la centrifugation sont rassemblées dans le surnageant qui est utilisé pour la détermination des activités libres.

Les activités totales sont mesurées après homogénéisation du muscle dans une solution aqueuse de Triton $\times$ Ioo à $0,2 \mathrm{p}$. Ioo et incubation de l'homogénat à $37^{\circ} \mathrm{C}$ pendant deux heures.

Deux activités d'origine lysosomale ont été mesurées, l'activité cathepsine $\mathrm{D}$ testée à $\mathrm{pH} 3.5$ sur de l'hémoglobine dénaturée et l'activité $\beta$-glucuronidase déterminée à $\mathrm{pH} 5$ avec le phénolphtaléine $\beta$-D glucuronide.

Les activités lacticodéshydrogénases totales et libres ont été également mesurées.

Dans la technique d'extraction décrite ci-dessus, la phase d'imbibition dans la solution de saccharose est limitée à deux heures. Pour des périodes d'imbibition plus longues, allant jusqu'à six heures on n'enregistre pas, après ultracenrifugation, d'augmentation de la quantité de protéines extraites ni des activités enzymatiques mesurées dans ces fractions. Pour vérifier si la fraction des protéines sarcoplasmiques obtenue après imbibition et ultracentrifugation permet de déterminer le niveau réel des activités enzymatiques libres, nous avons mesuré sur cette fraction l'activité LDH. Au temps 0,2 jours et 8 jours post mortem les activités LDH libres représentent en moyenne pour les 4 animaux 60,78 et 73 p. roo des activités LDH totales. Or, ces résultats corroborent ceux de Petre (1960) obtenus après extraction fractionnée d'un homogénat de muscle ainsi que ceux de Brody et Hengel (I964) obtenus par l'étude histochimique de la répartition des différents isoenzymes de la $\mathrm{LDH}$ du muscle.

Au cours de la période étudiée il n'y a pas eu de diminution des activités hydrolytiques totales tant pour la cathepsine D que pour la $\beta$-glucuronidase. Par contre, on constate une augmentation progressive de ces activités hydrolytiques au sein des protéines sarcoplasmiques solubles comme l'indique le tableau I qui rapporte l'évolution des activités spécifiques cathepsine $D$ et $\beta$-glucuronidase mesurées sur les protéines obtenues après imbibition et ultracentrifugation du muscle.

L'augmentation des activités spécifiques est la preuve d'une diffusion progressive dans le sarcoplasme de ces enzymes hydrolytiques.

TABLEAU I

Activités spécifiques libres cathepsine $D$ et $\beta$-glucuronidase exprimées respectivement en $\gamma$ tyrosine et $\gamma$ phénolphtaléine libérés par $m g$ de protéines et par heure

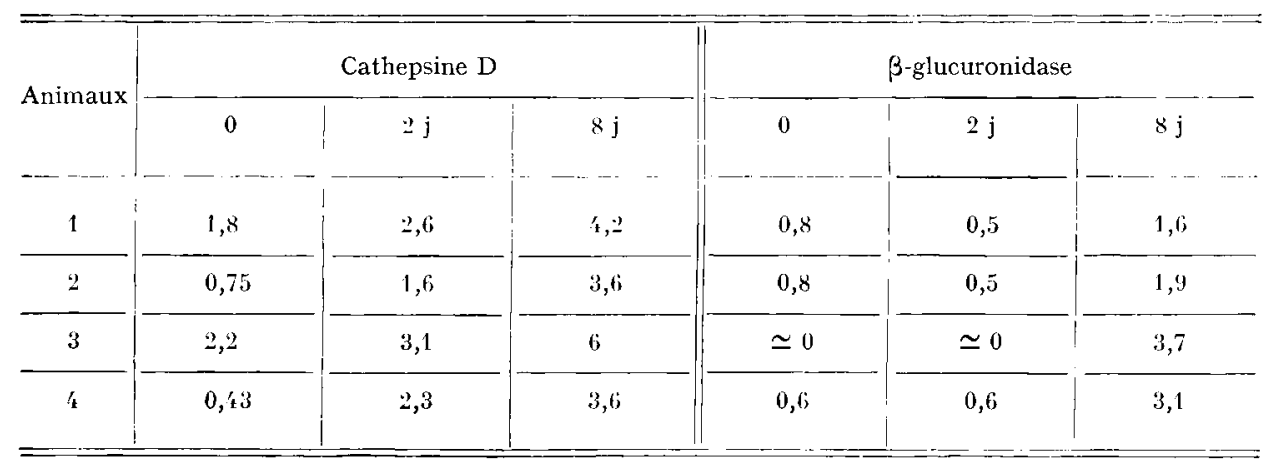


Cependant cette diffusion reste faible au cours de la période étudiée comme l'indiquent les figures I et 2 qui représentent l'évolution du pourcentage des activités libres par rapport aux activités totales entre le temps o et 8 jours post mortem.

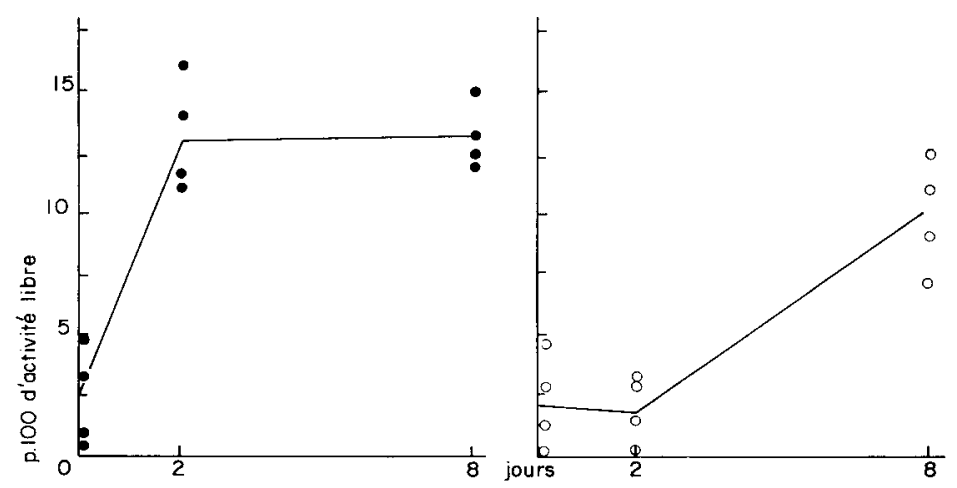

FIG. I. - Evolution post mortem de l'activité libre cathepsine $D$ en p. 100 de l'activité totale FIG. 2. - Evvolution post mortem de l'activité libre $\beta$-glucuronidase en p. 100 de l'activité totale

Ces résultats diffèrent de ceux de PARRISH ( 1967 ) qui observait un taux de libération très supérieur. Mais cet auteur recourait à une méthode classique d'extraction des protéines sarcoplasmiques par broyage et homogénéisation du muscle dans une solution de saccharose $0,25 \mathrm{M}$ tamponné à $\mathrm{pH} 7,4$. De même RAHman (1964) en travaillant avec le même milieu d'extraction sur la rate, le foie et le thymus de rat, après homogénéisation observait des activités libres variant de 20 à 50 p. Ioo de l'activité totale.

D'autre part on peut constater sur ces graphiques une différence très nette entre les cinétiques de libération des activités cathepsine $D$ et $\beta$-glucuronidase ce qui semble traduire que la libération in situ, de ces enzymes ne s'opère pas selon un mécanisme de tout ou rien comme le suppose l'école de Duve, (Beaufay, de Duve, 1959). Par contre, ce résultat corrobore celui de Sawant, Desal et Tappel (1964) obtenus après étude in vitro de suspension de lysosomes.

En conclusion on peut constater qu'au cours de la période post mortem étudiée, dans le muscle préservé de tout traitement mécanique le taux de libération des hydrolases acides reste très faible. Par contre, une simple congélation-décongélation entraîne une augmentation considérable des activités libres dont les taux dépassent $50 \mathrm{p}$. roo de l'activité totale pour l'activité cathepsine $\mathrm{D}$ et 30 p. roo pour l'activité $\beta$-glucuronidase, mesurée selon la méthode décrite ci-dessus. A la simple vue de ces résultats on pourrait conclure qu'un potentiel hydrolytique important est sous employé au cours de la maturation de la viande. S'il devait s'avérer que ces enzymes pourraient avoir une action au niveau des constituants de la structure du muscle, protéines myofibrillaires et constituants du tissu conjonctif, ce qui reste à prouver, une voie technologique à l'amélioration de la tendreté de la viande pourrait se concevoir par la mise en ouvre totale de ce système enzymatique. 


\section{SUMMARY}

\section{ACTIVATION OF LYSOSOMAI, ENZYMES IN BEEF MUSCLE DURING AGING OF MEAT}

The release of lyosomal enzymes in beef muscle during aging of meat was studied.

The free hydrolytic activities were measured in the sarcoplasmic proteins of press juice obtained by ultra centrifugation of muscle fragments in a slightly hypertonic sucrose solution buffered at $\mathrm{pH} 7.4$. This method was used to prevent the enzyme release which could have occured during homogeneization of the muscle. Cathepsin-D and $\beta$-glucuronidase activities were estimated. Free specific activity of both enzymes increased during aging of meat, but eight days post mortem the total free activities were still less than i 5 p. Ioo of the total activities.

The kinetics of cathepsin D and $\beta$-glucorunidase release are different. While the rigor processes, the free cathepsin-D activity increases, whereas the $\beta$-glucuronidase activity is released when the resolution of rigor begins.

\section{RÉFÉRENCES BIBLIOGRAPHIQUES}

Amberson W. R., Bauer A. C., Philpott D. E., Roisen F., ig64. Proteins and enzyme activities of press juices of white red and heart muscles of the rabbit. J. Cell Comp. Physiol., 63, 7-24.

Beaufay H., DE Duve C., 1959. Tissue fractionation studies. Enzyme release of bound hydrolases. Biochem. J., 73, 604-6II.

Bodwell C. E., Pearson A. M., I964. The activity of partially purified catheptic enzymes on various natural and synthetic substrats. J. Food Sci., 29, 602-606.

Brody I. A., ENGEL W. K., I964. Isozyme histochemistry. The display of selective lactate dehydrogenase isozymes in sections of skeletal muscle. J. Histochem. Cytochem., 12, 687-695.

Brunet-Martins C., Whitaker J. R., 1968, Catheptic enzymes and meat tenderization. J. Food Sci., 33, 59-69.

Gardner G. A., Stewart D. J., 1966. Changes in the free amino and other nitrogen compounds in stored beef muscle. J. Sci. Fd. A gric, 17, 49 I-496.

Lawrie R. A., Ig66. Meat Science. Pergamon Press.

McIntosi E. N., I967. Effect of post mortem aging and enzyme tenderizers on mucoprotein of bovin skeletal muscle. J. Food Sci., 32, 2 10-213.

Parrish F. C., I967. Hydrolytic enzyme of porcine muscle. J. Anim. Sci., 26, 899.

Pette I), I960. In R. Czok T. Bücher. Crystallized enzymes from the myogen of rabbit skeletal muscle. Adv. Protein Chem., 15, 315-4I5.

Rahman Y. E., 1964. A note on acid phosphatase release from spleen liver and thymus of rats. Bioch. Biophys. acta, 90, 440-442.

Sawant P. L., Desai I. D., Tappel A. L., I964. liactors affecting the lysosomal membrane and availability of enzymes. Arch. Bioch. Biophys., 105, 247-253.

Suzuki A., Nakazato M., I'ujimaki M., 1967. Studies on proteolyses in stored muscle. Agric. Biol. Chem., 31, 953-957. 